\title{
MUTATIONS IN THE QUINOLONE RESISTANCE-DETERMINING REGIONS OF GYRA AND PARC IN ENTEROBACTERIACEAE ISOLATES FROM BRAZIL
}

\author{
Luciene A. R. Minarini ${ }^{1,2}$, Ana Lucia C. Darini ${ }^{{ }^{*}}$ \\ ${ }^{1}$ Departamento de Análises Clínicas, Toxicológicas e Bromatológicas, Faculdade de Ciências Farmacêuticas de Ribeirão Preto, \\ Universidade de São Paulo, Ribeirão Preto, SP, Brasil; ${ }^{2}$ Instituto de Ciências Ambientais, Químicas e Farmacêuticas, \\ Universidade Federal de São Paulo, Diadema, SP, Brasil.
}

Submitted: May 25, 2011; Returned to authors for corrections: January 12, 2012; Approved: June 07, 2012.

\begin{abstract}
Mutations in the quinolone resistance-determining regions (QRDR) in chromosomal gyrA and parC genes and fluoroquinolone susceptibility profiles were investigated in quinolone-resistant Enterobacteriaceae isolated from community and hospitalized patients in the Brazilian Southeast region. A total of 112 nalidixic acid-resistant enterobacterial isolates collected from 2000 to 2005 were investigated for mutations in the topoisomerases genes gyrA and parC by amplifying and sequencing the QRDR regions. Susceptibility to fluoroquinolones was tested by the agar dilution method. Amongst the 112 enterobacterial isolates, 81 (72.3\%) were resistant to ciprofloxacin and 5 (4.5\%) showed reduced susceptibility. Twenty-six $(23.2 \%)$ were susceptible to ciprofloxacin. Several alterations were detected in gyrA and parC genes. Escherichia coli isolates (47.7\%) showed double mutations in the gyrA gene and a single one in the parC gene. Two unusual aminoacid substitutions are reported, an Asp87-Asn in a Citrobacter freundii isolate with reduced susceptibility to fluoroquinolones and a Glu84-Ala in one E. coli isolate. Only a parC gene mutation was found in fluoroquinolone-susceptible Enterobacter aerogenes. None of the isolates susceptible to ciprofloxacin presented mutations in topoisomerase genes. This comprehensive analysis of QRDRs in gyrA and parC genes, covering commonly isolated Enterobacteriaceae in Brazil is the largest reported up to now.
\end{abstract}

Key words: quinolone resistance-determining region, mutations, gyrA, parC, Enterobacteriaceae.

\section{INTRODUCTION}

Fluoroquinolones have been frequently prescribed as empirical therapy against most hospital and community infections due to increased appearance of multiple drug resistant Gram-negative pathogens and to the disease severity $(2,8,22)$. In countries with extensive clinical use of quinolones, fluoroquinolone resistance has been a problem in clinical medicine for its limiting of available agents in the treatment of many types of infection $(1,7,15,23)$.

Brazil has one of the highest rates of resistance to quinolones among Latin American countries. A report from the SENTRY Antimicrobial Surveillance Program revealed that resistance to nalidixic acid was higher in Latin American

*Corresponding Author. Mailing address: Departamento de Análises Clínicas, Toxicológicas e Bromatológicas, Faculdade de Ciências Farmacêuticas de Ribeirão Preto, Universidade de São Paulo, USP, Av. do Café s/non, 14040-903, Ribeirão Preto.; Tel.: +55 16 3602-4291 Fax: +55 16 3602-4725.; E-mail: aldarini@,fcfrp.usp.br 
(15\%) than in North America (6.3\%), being as high as $50 \%$ in Mexico and $33.6 \%$ in Brazil (1). Brazilian investigators confirmed the information reporting that the significant increase in resistance to quinolones detected in Brazilian medical centers between 2002-2006 may be related to increased drug use in community acquired infections and hospital-wide antibiotic pressure $(9,18)$.

Fluoroquinolone resistance has been associated with DNA gyrase and topoisomerase IV alterations for both Gramnegative and Gram-positive organisms. Structural topoisomerase changes reducing the affinity of this enzyme to fluoroquinolones are caused by mutations in the quinolone resistance-determining regions (QRDR) of gyrA and parC genes $(14,21)$. Other mechanisms involve mutations affecting the intracellular accumulation of fluoroquinolones in the cell wall (19) and the expression of outer membrane proteins (OMP) leading to reduced entry of antibiotics $(17,24)$. Three additional mechanisms of quinolone resistance have been reported. These refer to the presence of Qnr proteins, which protect type II topoisomerases from quinolones $(10,11,16)$; of a variant aminoglycoside acetyltransferase, $\operatorname{Aac}\left(6^{\prime}\right)-\mathrm{Ib}-\mathrm{cr}$, that modifies ciprofloxacin (20) and of active drug efflux pumps QepA and OqxAB, belonging to the major facilitator superfamily (MFS) transporters (6, 27). Topoisomerase mutations have not been, up to now, systematically investigated and explored in clinical isolates from Brazil, except in a report on ciprofloxacin susceptible E. coli (5).

The objective of this study was to assess the presence of mutations in the QRDR of chromosomal gyrA and parC genes and fluoroquinolone susceptibility profile in quinoloneresistant Enterobacteriaceae isolated from community and hospital patients in the Brazilian Southeast region.

\section{MATERIALS AND METHODS}

\section{Bacterial isolates and susceptibility testing}

From 2000 to 2005, 112 clinical non-duplicate nalidixic acid- resistant Enterobacteriaceae isolates were obtained from outpatients $(n=91)$ and inpatients $(n=21)$ referred to laboratories located in the city of Juiz de Fora (State of Minas Gerais ) and Ribeirão Preto (State of São Paulo ). All species were identified both by conventional techniques and by MiniAPI, a semi-automatized assay (bioMérieux, Marcy l'Etoile, France). Susceptibility to fluoroquinolones was tested by the agar dilution method with E. coli ATCC 25922 as a control reference in MIC determinations. Susceptibility and resistance breakpoints were those defined by the Clinical and Laboratory Standards Institute (3).

\section{Detection of mutations in the topoisomerase genes}

DNA extraction and PCR analysis were performed as described elsewhere using sets of primers designed for amplification and sequencing of topoisomerase genes (10). DNA was amplified using an initial denaturation step of $5 \mathrm{~min}$ at $94^{\circ} \mathrm{C}$, followed by 30 cycles consisting of $1 \mathrm{~min}$ at $94^{\circ} \mathrm{C}, 1$ min at the annealing temperature of $55^{\circ} \mathrm{C}$ and $1 \mathrm{~min}$ at $72^{\circ} \mathrm{C}$, and a final extension step of $10 \mathrm{~min}$ at $72^{\circ} \mathrm{C}$. Amplified DNA products were resolved by electrophoresis in 1\% agarose gels and staining with ethidium bromide. PCR products were purified for sequencing by $\mathrm{GFX}^{\mathrm{TM}}$ PCR (GE Healthcare, Buckinghamshire, UK). The forward and reverse strands were sequenced in MegaBACE 1000 (GE Healthcare) with the same PCR primer sets. Raw sequences were reviewed by visual inspection using the software Chromas v.1.45 (32-bit) (Technelysium Pty. Ltd). QRDR nucleotide sequences in gyrA and parC genes from each of the 112 isolates were compared with the respective reference sequences in the GenBank database (accession numbers from AF052253 to AF052260).

\section{RESULTS AND DISCUSSION}

Species distribution among the 112 Enterobacteriaceae isolates was: Escherichia coli $(\mathrm{n}=65)$, Klebsiella pneumoniae $(\mathrm{n}=21)$, Klebsiella oxytoca $(\mathrm{n}=12)$, Enterobacter cloacae $(\mathrm{n}=6)$, Citrobacter freundii $(\mathrm{n}=2)$, Providencia stuartii $(\mathrm{n}=2)$, Serratia marcescens $(\mathrm{n}=2)$, Enterobacter aerogenes $(\mathrm{n}=1)$ and Proteus 
mirabilis $(\mathrm{n}=1)$. Twenty-three percent of the 112 nalidixic acidresistant isolates were susceptible to ciprofloxacin and/or levofloxacin (MIC $\leq 1 \mu \mathrm{g} / \mathrm{mL}$ ).

Table 1 summarizes codon numbers and the corresponding alteration detected in the gyrA and parC genes of fluoroquinolone-resistant and susceptible clinical isolates included in the study and the corresponding MIC values. All Enterobacteriaceae isolates had amplification products.

Table 1. Fluoroquinolone MIC values, and alterations detected in the gyrA and parC genes of Enterobacteriaceae isolates

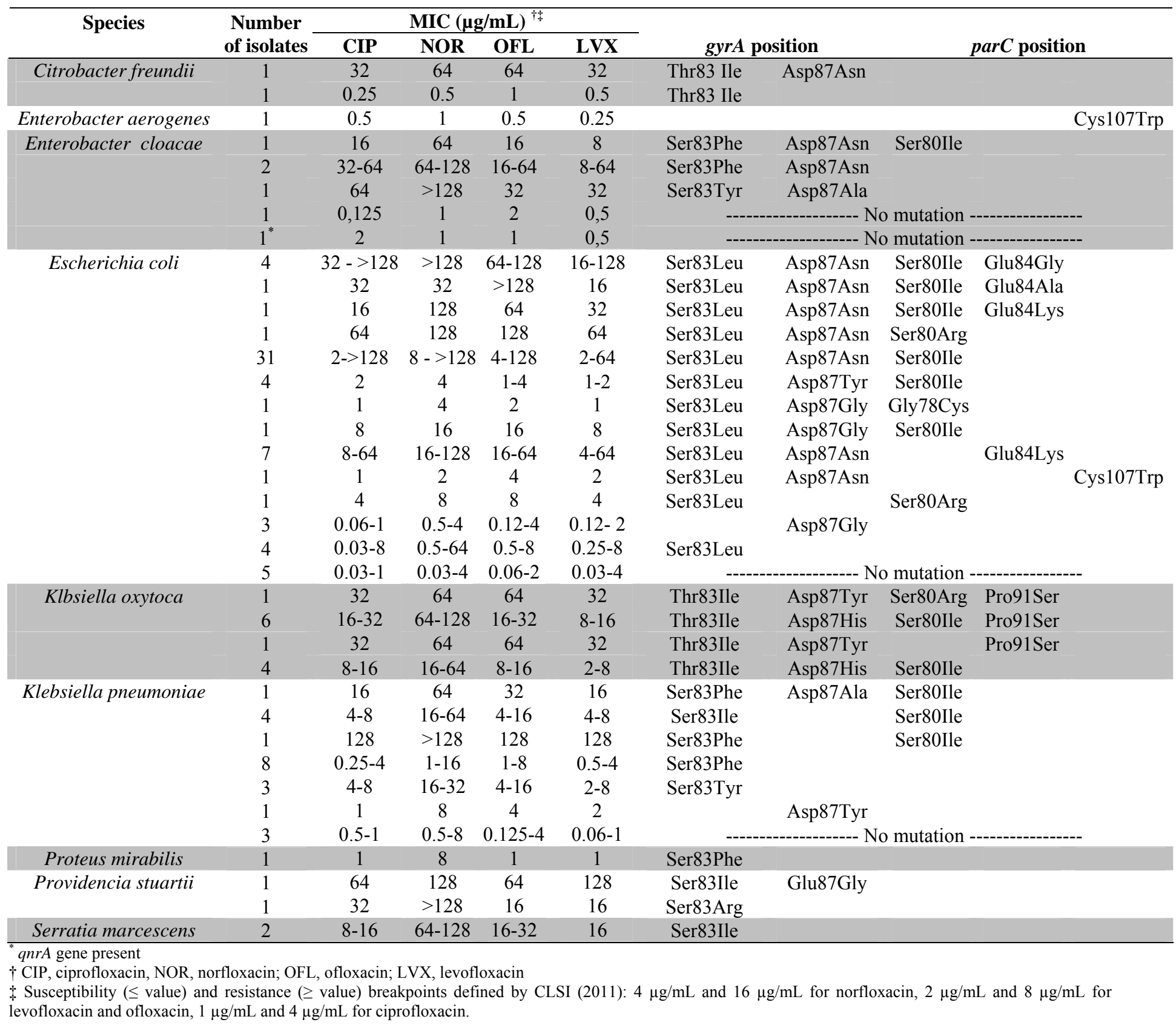

According to Ruiz (21), codons 83 and 87 in gyrA and codons 80 and 84 in parC gene display the most common alterations in clinical isolates. In addition, expression of high- level fluoroquinolone resistance in Enterobacteriaceae requires the presence of multiple mutations in gyrA and/or parC genes.

Topoisomerase genes in nine isolates susceptible to 
ciprofloxacin (MICs $\leq 1 \mu \mathrm{g} / \mathrm{mL}$ ) did not show mutations. However, one E. cloacae exhibiting decreased susceptibility to fluoroquinolones (MIC $2 \mu \mathrm{g} / \mathrm{mL}$ ) and no mutation in topoisomerase genes had a previously detected qnrA gene (4, 12).

The majority of Enterobacteriaceae isolates in the study (91\%) showed a QRDR region with mutations in codons 83 and/or 87 of the gyrA gene. Twelve different types of mutations were found amongst the collection analyzed. They were Thr83-Ile and Asp87-Asn in C. freundii, Ser83-Phe, Ser83-Tyr, Asp87-Asn and Asp87-Ala in E. cloacae, Ser83Leu, Asp87-Asn, Asp87-Tyr and Asp87-Gly in E. coli, Thr83Ile, Asp87-Tyr and Asp87-His in K. oxytoca, Ser83-Phe, Ser83-Tyr, Asp87-Ala and Asp87-Tyr in K. pneumoniae, Ser83-Phe in P. mirabilis, Ser83-Ile, Ser83-Arg and Glu87-Gly in P. stuartii and Ser83-Ile in S. marcescens (Table 1). All fluoroquinolone resistant E. coli isolates had the usual Ser83Leu substitution in gyrA gene but according to Vila et al. (25), the additional mutation in codon 87 is associated with an increased fluoroquinolone resistance. This was confirmed by the results for E. coli (Table 1), as four of the isolates with a single alteration, Ser83-Leu, had MICs $\leq 8 \mu \mathrm{g} / \mathrm{mL}$ for ciprofloxacin. However, the isolates with an additional and common mutation in codon 87(Asp87-Asn), as shown by $91.6 \%$ of the samples, had higher ciprofloxacin MICs $\geq 8$ $\mu \mathrm{g} / \mathrm{mL}$. Eleven K. pneumoniae isolates with a single alteration in codon 83 also showed ciprofloxacin MICs $<8 \mu \mathrm{g} / \mathrm{mL}$. Three $E$. coli isolates and one $K$. pneumoniae having a single alteration in codon 87, Asp87-Gly or Asp87-Tyr, had MIC $\leq 1$ $\mu \mathrm{g} / \mathrm{mL}$ for ciprofloxacin.

Codons 78, 80, 84 and 107 showed alterations in the QRDR of the parC gene. The replacements were Gly78-Cys, Ser80-Ile, Ser80-Arg, Glu84-Gly, Glu84-Ala, Glu84-Lys, Cys107-Trp in E. coli, Ser80-Ile in E. cloacae, Cys107-Trp in E. aerogenes, Ser80-Arg, Ser80-Ile, Pro91-Ser, in K. oxytoca and Ser80-Ile in K. pneumoniae. Analysis of the data presented in Table 1, did not indicate that these changes could be by themselves responsible for the difference in fluoroquinolone
MIC values among the different isolates.

A different substitution, not reported to date, at codon 84 of the parC gene (glutamine replaced by alanine) was exhibited by one E. coli isolate with Ser83-Leu, Asp87-Asn and Ser80Ile replacements. C. freundii, P. mirabilis, P. stuartii, $S$. marcescens isolates did not present mutations in parC gene.

Substitutions in gyrA and parC genes were found in $95.8 \%$ of fluoroquinolones-resistant $E$. coli isolates in this study. According to Table 1, 47.7\% of E. coli isolates showed substitutions: Ser83-Leu and Asp87-Asn in gyrA gene and Ser80-Ile in parC gene, while six isolates (9.2\%) (CIP MIC $\geq 16 \mu \mathrm{g} / \mathrm{mL}$ ) had Ser83-Leu and Asp87-Asn substitutions in gyrA gene, and Ser80-Ile and Glu84-Gly, Glu84-Ala or Glu84Lys in parC gene. Another uncommon substitution (serine by arginine) at codon 80 in parC gene was observed in one E. coli isolate. In general, a substitution in Ser-83 is sufficient to generate a high level of resistance to nalidixic acid, whereas a second mutation at Asp-87 in gyrA gene may play a complementary role in the development of high levels of ciprofloxacin resistance.

According to Weigel et al. (26), C. freundii isolates with reduced susceptibility to fluoroquinolones exhibited Thr83 to Ile mutations as a result of C-to- $\mathrm{T}$ substitutions at the nucleotide 248th position in gyrA. In one $C$. freundii isolate, in this study, another different substituted amino acid was detected in gyrA gene, Asp87-Asn instead of glycine, which to our knowledge has not been described so far. Although rarely reported in the literature, alterations in codon 83 (serine for isoleucine) and 87 (asparagine for alanine or tyrosine) in gyrA gene were detected in $K$. pneumoniae isolates $(4,26)$. No such alteration in the parC gene of the ciprofloxacin susceptible $K$. pneumoniae was found.

Just one alteration, Thr83-Ile, in gyrA gene was reported by Weigel et al. (26) in ciprofloxacin resistant K. oxytoca. Additional substitutions were found in this study, Asp87-Tyr in 2 isolates and Asp87-His in 10 others having MICs for ciprofloxacin of $32 \mu \mathrm{g} / \mathrm{mL}$ and $8-16 \mu \mathrm{g} / \mathrm{mL}$ respectively. All fluoroquinolone resistant $K$. oxytoca isolates had mutations 
detected in gyrA and parC genes, the most frequent being Ser80-Ile and Pro91-Ser. The alterations found in P. stuartii and $S$. marcescens isolates were the same reported by Weigel et al. (26).

It has been shown that the primary quinolone target in Enterobacteriaceae is gyrase and topoisomerase IV enzymes, since mutations in parC or parE are observed only in addition to the ones in DNA gyrase (7). However, the only fluoroquinolone susceptible E. aerogenes tested had just one mutation in codon 107 in parC gene, Cys107-Trp.

A diversity of alterations has been found in gyrA and parC genes, but there was not a clear correlation between frequency of distribution of each alteration type and fluoroquinolone MIC values, which varied from 2 to $>128 \mu \mathrm{g} / \mathrm{mL}$ for ciprofloxacin, from 4 to $128 \mu \mathrm{g} / \mathrm{mL}$ for ofloxacin and from 2 to $64 \mu \mathrm{g} / \mathrm{mL}$ for levofloxacin.

Consistent with previous studies, low-level fluoroquinolone resistance in E. coli is associated with a single alteration in the GyrA protein while high-level resistance required double mutations $(2,23)$. However, in all other species in this study, there were isolates exhibiting a single alteration in gyrA gene and high-level fluoroquinolone resistance. Other mechanisms implicated in fluoroquinolone resistance, which might be responsible for the differences in MICs values for fluoroquinolones observed among the isolates with the same types of mutation in all genes were not considered in the present study. Changes in permeability and active efflux are mechanisms that cannot be excluded and may enhance development of resistance and contribute to the selection of fluoroquinolone-resistant isolates in the course of treatments with these antibiotics. Furthermore, the presence of one or more related clones in the collection studied was not determined by molecular typing methodologies.

In conclusion, this study documented alterations in the QRDR segments of gyrA and parC genes in ciprofloxacin susceptible and resistant Enterobacteriaceae recovered from clinical specimens in the Southeast region of Brazil. The alterations reported are the most detailed in the QRDR in gyrA and parC genes and include the ones in commonly isolated Enterobacteriaceae. Additional data on topoisomerase sequences and mutations associated with fluoroquinolone resistance in clinical isolates of diversified Enterobacteriaceae species was also provided.

\section{ACKNOWLEDGEMENTS}

The authors are grateful to Lemos Laboratórios de Análises Clínicas, Juiz de Fora (Minas Gerais State) and University Hospital of Ribeirão Preto, Ribeirão Preto Campus of the University of São Paulo for supplying the isolates; to Izabel C. V. Palazzo for assistance in MIC determinations; to the Laboratory of Molecular Genetics and Bioinformatics, Department of Genetics, Faculty of Medicine of Ribeirão Preto, University of São Paulo for sequencing assays. This study was financially supported by Fundação de Amparo à Pesquisa do Estado de São Paulo (FAPESP Proc n. 2006/02320-9) and Coordenação de Aperfeiçoamento de Pessoal de Nível Superior (CAPES), Brazil.

\section{REFERENCES}

1. Biedenbach, D.J.; Toleman, M.; Walsh, T.R.; Jones, R.N. (2006). Analysis of Salmonella spp. with resistance to extended-spectrum cephalosporins and fluoroquinolones isolated in North America and Latin America: report from the SENTRY Antimicrobial Surveillance Program (1997-2004). Diagn. Microbiol. Infect. Dis. 54 (1), 13-21.

2. Chenia, H.Y.; Pillay, B.; Pillay, D. (2006). Analysis of the mechanisms of fluoroquinolone resistance in urinary tract pathogens. J. Antimicrob. Chemother. 58 (6), 1274-1278.

3. CLSI (2011). Performance standards for antimicrobial susceptibility testing; Twenty-first informational supplement Document M100-S21. Wayne, Pa: Clinical and Laboratory Standards Institute.

4. Deguchi, T.; Yasuda, M.; Nakano, M.; Ozeki, S.; Kanematsu, E.; Nishino, Y.; Ishihara, S.; Kawada, Y. (1997). Detection of mutations in the gyrA and parC genes in quinolone-resistant clinical isolates of Enterobacter cloacae. J. Antimicrob. Chemother. 40 (4), 543-549.

5. Gales, A.C.; Gordon, K.A.; Wilke, W.W.; Pfaller, M.A.; Jones, R.N. (2000). Occurrence of single-point gyrA mutations among ciprofloxacinsusceptible Escherichia coli isolates causing urinary tract infections in Latin America. Diagn. Microbiol. Infect. Dis. 36 (1), 61-64. 
6. Hansen, L.H.; Jensen, L.B.; Sørensen H.I.; Sørensen S.J. (2007). Substrate specificity of the OqxAB multidrug resistance pump in Escherichia coli and selected enteric bacteria. J. Antimicrob. Chemother. 60(1), 145-147.

7. Hooper, D.C. (2002). Fluoroquinolone resistance among Gram-positive cocci. Lancet Infect. Dis. 2 (9), 530-538.

8. Hurst, M.; Lamb, H.M.; Scott, L.J.; Figgitt, D.P. (2002). Levofloxacin: an updated review of its use in the treatment of bacterial infections. Drugs 62 (14), 2127-2167.

9. Jacoby, T.S.; Kuchenbecker, R.S.; Dos Santos, R.P.; Magedanz, L.; Guzatto, P.; Moreira, L.B. (2010). Impact of hospital-wide infection rate, invasive procedures use and antimicrobial consumption on bacterial resistance inside an intensive care unit. J. Hosp. Infect. 75 (1), 23-27.

10. Mammeri, H.; Van De Loo, M.; Poirel, L.; Martinez-Martinez, L.; Nordmann, P. (2005). Emergence of plasmid-mediated quinolone resistance in Escherichia coli in Europe. Antimicrob. Agents Chemother. 49 (1), 71-76.

11. Martinez-Martinez, L.; Pascual, A.; Jacoby, G.A. (1998). Quinolone resistance from a transferable plasmid. Lancet 351 (9105), 797-799.

12. Minarini, L.A.; Gales, A.C.; Darini, A.L. (2007). First report of plasmidmediated resistance to quinolones and cefotaxime in an Enterobacter cloacae isolated from an outpatient in Brazil. Antimicrob. Agents Chemother. 51 (1), 401-402.

13. Minarini, L.A.; Gales, A.C.; Palazzo, I.C.; Darini, A.L. (2007). Prevalence of community-occurring extended spectrum beta-lactamaseproducing Enterobacteriaceae in Brazil. Curr. Microbiol. 54 (5), 335341.

14. Moon, D.C.; Seol, S.Y.; Gurung, M.; Jin, J.S.; Choi, C.H.; Kim, J.; Lee, Y.C.; Cho, D.T.; Lee, J.C. (2010). Emergence of a new mutation and its accumulation in the topoisomerase IV gene confers high levels of resistance to fluoroquinolone in Escherichia coli isolates. Int. J. Antimicrob. Agents 35 (1), 76-79.

15. Naheed, A.; Kalluri, P.; Talukder, K.A.; Faruque, A.S.; Khatun, F.; Nair, G.B.; Mintz, E.D.; Breiman, R.F. (2004). Fluoroquinolone-resistant Shigella dysenteriae type 1 in northeastern Bangladesh. Lancet Infect. Dis. 4 (10), 607-608.

16. Nordmann, P.; Poirel, L. (2005). Emergence of plasmid-mediated resistance to quinolones in Enterobacteriaceae. J. Antimicrob. Chemother. 56 (3), 463-469.

17. Ogbolu, D.O.; Daini, O.A.; Ogunledun, A.; Alli, A.O.; Webber, M.A. (2010). High levels of multidrug resistance in clinical isolates of Gram- negative pathogens from Nigeria. Int. J. Antimicrob. Agents 37 (1), 6266.

18. Pereira, A.S.; Andrade, S.S.; Monteiro, J.; Sader, H.; Pignatari, A.C.C.; Gales, A.C. (2007). Evaluation of the susceptibility profiles, genetic similarity and presence of $q n r$ gene in Escherichia coli resistant to ciprofloxacin isolated in Brazilian hospitals. Braz. J. Infect. Dis. 11 (1), 40-43.

19. Poole, K. (2005). Efflux-mediated antimicrobial resistance. J. Antimicrob. Chemother. 56 (1), 20-51.

20. Robicsek, A.; Sahm, D.F.; Strahilevitz, J.; Jacoby, G.A.; Hooper, D.C. (2005). Broader distribution of plasmid-mediated quinolone resistance in the United States. Antimicrob. Agents Chemother. 49 (7), 3001-3003.

21. Ruiz, J. (2003). Mechanisms of resistance to quinolones: target alterations, decreased accumulation and DNA gyrase protection. $J$. Antimicrob. Chemother. 51 (5), 1109-1117.

22. Tumbarello, M.; Trecarichi, E.M.; Bassetti, M.; De Rosa, F.G.; Spanu, T.; Di Meco, E.; Losito, A.R.; Parisini, A.; Pagani, N.; Cauda, R. (2011). Identifying patients harboring ESBL-Producing Enterobacteriaceae on hospital admission: Derivation and validation of a scoring system. Antimicrob. Agents Chemother. (May 2).

23. Vernaz, N.; Huttner, B.; Muscionico, D.; Salomon, J.L.; Bonnabry, P.; López-Lozano, J.M.; Beyaert, A.; Schrenzel, J.; Harbarth, S. (2011). Modelling the impact of antibiotic use on antibiotic-resistant Escherichia coli using population-based data from a large hospital and its surrounding community. J. Antimicrob. Chemother. 66 (4), 928-935.

24. Vila, J.; Ruiz, J.; Marco, F.; Barcelo, A.; Goñi, P.; Giralt, E.; Jimenez de Anta, T. (1994). Association between double mutation in gyrA gene of ciprofloxacin-resistant clinical isolates of Escherichia coli and MICs. Antimicrob. Agents Chemother. 38 (10), 2477-2479.

25. Vila, J.; Ruiz, J.; Navia, M.M. (1999). Molecular bases of quinolone resistance acquisition in Gram-negative bacteria. In: S.G. Pandaly, editor. Recent Res Devel Antimicrob Agents Chemother. India: Reseach Signpost; p. 323-344.

26. Weigel, L.M.; Steward, C.D.; Tenover, F.C. (1998). gyrA mutations associated with fluoroquinolone resistance in eight species of Enterobacteriaceae. Antimicrob. Agents Chemother. 42 (10), 2661-2667.

27. Yamane, K.; Wachino, J.; Suzuki, S.; Kimura, K.; Shibata, N.; Kato, H.; Shibayama, K.; Konda, T.; Arakawa, Y. (2007). New Plasmid-Mediated Fluoroquinolone Efflux Pump, QepA, Found in an Escherichia coli Clinical Isolate. Antimicrob. Agents Chemother. 51 (9), 3354-3360. 